\title{
Thematic Mapping: This Is My Truth Tell Me Yours
}

\author{
Kenneth Field ${ }^{\mathrm{a}, *}$ \\ a EsriInc,kfield@esri.com \\ * Corresponding author
}

Keywords: education, cartography book, MOOC

\begin{abstract}
:
How many maps can you make using a single thematic dataset? One? Ten? A hundred? More...? It turns out there's not really an answer and yet the answer you may have provided will be determined by many different influences. You may be a trained cartographer and, so your immediate reaction will have likely been 'quite a few' but without fixating on a specific number. You may be new to map-making and simply don't know, even, if it's a trick question. Is there a finite number? You may use a particular software product and are guided by the number of options available to you out-of-thebox. Or, perhaps you have a very clear map in mind for a given dataset.
\end{abstract}

So let's expand the question a bit. How many maps can you make of the results of the 2016 United States Presidential election contested by Donald J. Trump and Hillary R. Clinton? Does that make the choice any clearer? Well that's the task of this presentation. And the answer, while not being infinite, is that there are likely as many choices you can make in selecting a map type and then designing it as you can imagine. And that's the job of a cartographer whose specialist expertise is to assess a dataset and then deliberate over how to map it to convey some aspect of its character to an audience. And all of those decisions are mediated by various contexts. Who is the map for? (general audience or partisan readership?) What type of medium will be used (digital or print? for a cellphone or a newspaper?). How big should the map be? Will it be constrained in any way by that? And do you want a map that shows incredible detail or an overview? Or is it designed to relay the results empirically or, perhaps be used simply to grab attention? More questions!

President Trump used the map in Figure 1 to report on his own victory. During one of his first press conferences, Reuters quotes Trump as saying "Here, you can take that, that's the final map of the numbers. It's pretty good, right? The red is obviously us."

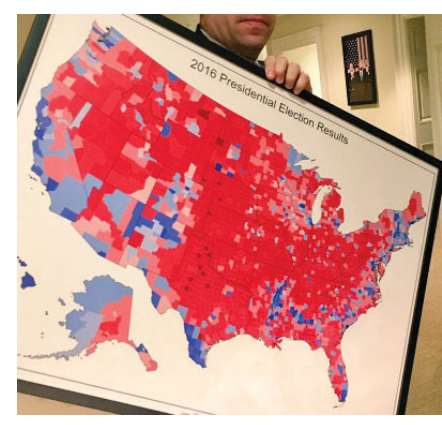

Yet Trump's map was roundly criticized (mostly by non-Republicans) as being a fake map. It presented a somewhat biased view of the results with huge swathes of red being used to promote the idea that victory was garnered from far and wide. More red gives the impression. Yet the map focuses very much on the geography of the United States which has a hugely dispersed population with large areas very sparsely populated and many highly populated places being seen relatively smaller on the map. Red naturally dominates this particular view yet it speaks to Trump's truth and is exactly the map to use to deliver his view. Had Clinton won, there would have been a very different looking map built from the same data yet persuading us of how blue victory was. Trump's victory was marginal. Clinton won the popular vote but that's irrelevant because that's not how the result of the American democratic voting system is counted.

Figure 1. Trump's map

Quoting former British Prime Minister Harold Wilson: "a week is a long time in politics". The same might be said about electoral cartography. For many, elections provide a fascinating sideshow in seeing how the results are handled cartographically. In framing the presentation, I'll use recent United Kingdom elections to briefly review shifts in cartographic style and the emergence of a fascinating consensus in terms of map type, style and functionality. I'll also explore some of the maps from the 2016 Presidential election that we saw across the media. The geographies of two massively different countries account for some differences in approach but consumer preference also creates different demands in the map reader. Approaches range from the purely functional to beautifully imaginative and innovative artistic representations. I finish by sharing my own attempts to map recent political events, both artistically and to challenge and extend the palette of political thematic cartography.

I'll then present some original work that uses the 2016 Presidential election data to provide a way of looking at thematic mapping. The benefit of using a single dataset is that it gives an immediate visual comparison across the many different maps. It gives a baseline for understanding how the maps differ and provides an accessible catalogue of design choices for people to use as a guide to mapping in different, interesting and compelling ways. Throughout, I'll explore many of the small decisions that a cartographer might consider in their choices because each map type brings with it a range of benefits, drawbacks and aspects to consider, and these all play a major role in what your map will end up looking like and how it will be read and interpreted. 
I provide a catalogue of options showcasing the 2016 Presidential election results (Figure 2). Election data provide an extremely rich source of opportunities to underpin the maps to be made and a great way to demonstrate how cartography plays a critical role in the different truths that can be told. Very few, if any, maps are 'right or wrong' but they all tell shades of a different truth and speak to different agendas.

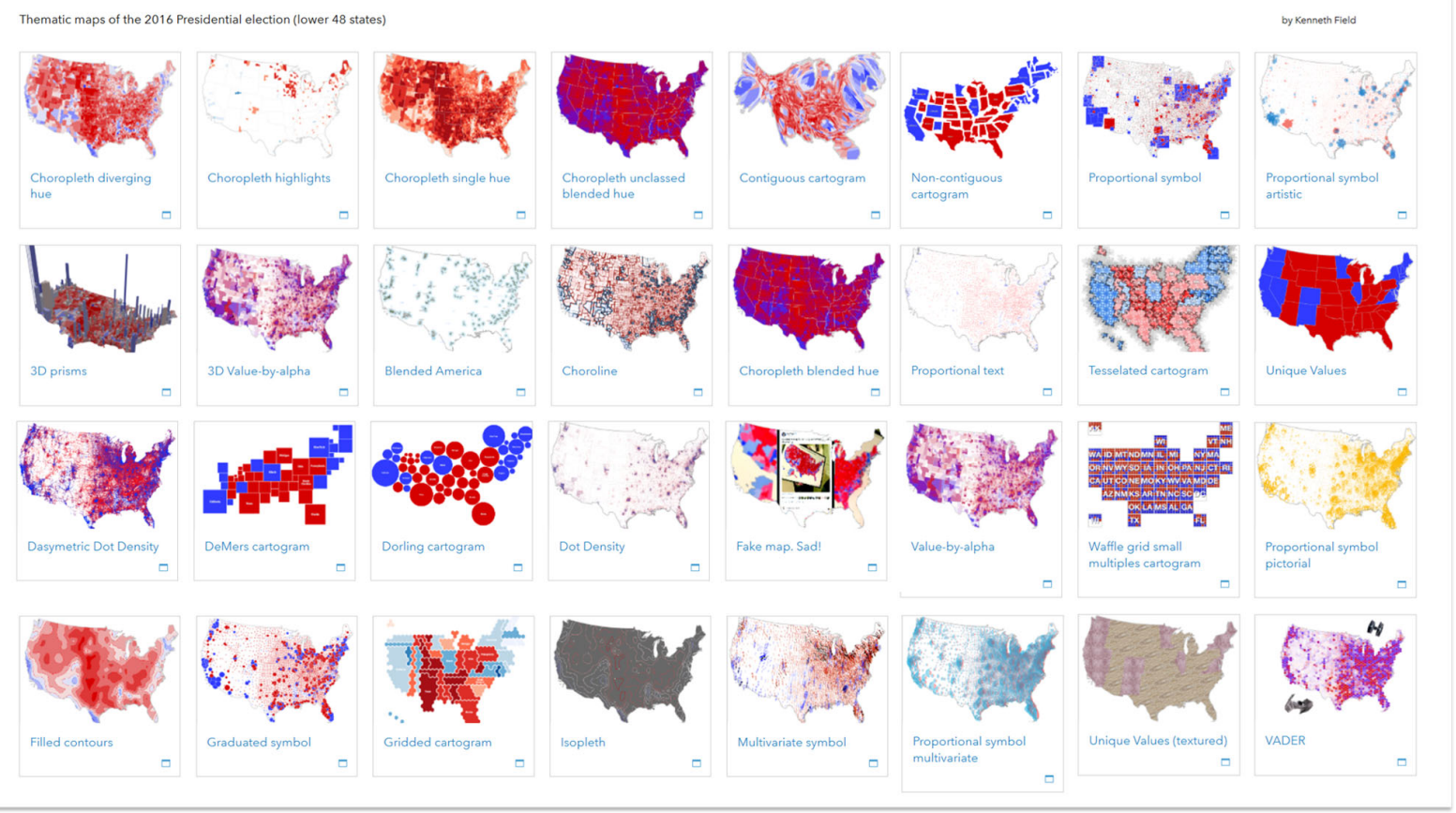

Figure 2: A gallery of thematic maps for the 2016 Presidential election

This aspect is critical to understanding how to match your maps to your story. With that in mind, of course, some maps will speak more to a Republican agenda, some more to a Democrat agenda and some would be seen as more neutral. That's inevitable as there are as many ways to make a persuasive, partisan map as there are an objective map. And what of a map's objectivity anyway? Maps are rarely made outside of a system that involves human input and while we might like to think that our maps are objective, our very involvement brings subjectivity to the party. Learning how to control subjective tendencies, manage our personal influences and make clear judgements can help you not only tell a better story through your map, but also limit the potential for your map to be seen as politically charged or partisan. Unless, of course, that is what you set out to do in the first place.

Maps, then, are tangible objects that add stature to debates, poll results, and the reporting of results which give them a sense of realism where perhaps one should not be presumed to yet exist. They report some aspect. That's as much as they can ever do. And they can be portrayed in different ways so the map reader has to be aware of the possible biases or uncertainties inherent in any map, not just political ones.

Maps also give newspapers, web and broadcast media (as well as political commentators) a way to flex their technological and design muscles in a game of carto-one-up-manship. We often see some fascinating and innovative cartography used in reporting election results. People's fascination with the picture of the results is experienced through the cartographies used and, often, the more dramatic the image, the more attention it gets. Maps are a battleground in their own right and used as a way to lure viewers to their coverage, to support their version of the truth as opposed to a competitor's truth, as much as they are simply a vehicle to report the results. What is truth anyway though? As far as electoral cartography goes, there are many different shades of the truth. 